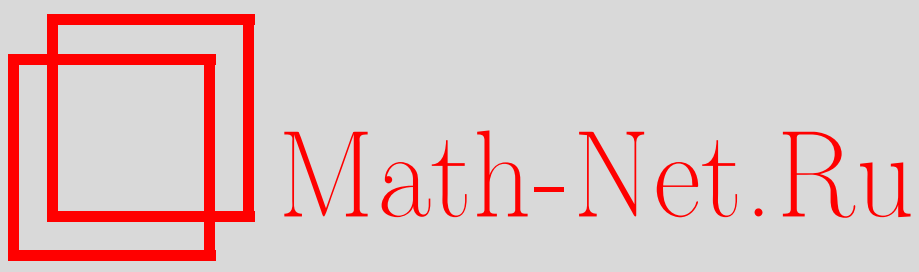

М. И. Дьяченко, Коэффициенты Фурье кусочно монотонных функций многих переменных, Изв. РАН. Сер. матем., 1998, том 62, выпуск 2, 35-48

DOI: https://doi.org/10.4213/im172

Использование Общероссийского математического портала Math-Net.Ru подразумевает, что вы прочитали и согласны с пользовательским соглашением

http://www . mathnet.ru/rus/agreement

Параметры загрузки:

IP : 54.164 .48 .24

26 апреля 2023 г., 12:06:09 
УДК 517.52

М.И. Дьяченко

\section{Коэффициенты Фурье кусочно монотонных функций многих переменных}

Основным результатом статьи является получение окончательной оценки для сумм Харди-Литтлвуда коэффициентов Фурье кусочно монотонных функций из пространств $L_{p}\left([-\pi, \pi)^{m}\right)$ при $2<p<2+\frac{1}{m-1}$.

Библиография: 7 наименований.

\section{§1. Введение}

Пусть $m$ - натуральное число, $T=[-\pi, \pi), \mathbb{R}_{+}=(0, \infty)$, а $\mathbb{Z}^{m}$ и $\mathbb{Z}_{+}^{m}$ суть целочисленные решетки в $\mathbb{R}^{m}$ и $\mathbb{R}_{+}^{m}$ соответственно. Если $\mathbf{x}, \mathbf{y} \in \mathbb{R}^{m}$, то обозначим:

$$
\begin{gathered}
\mathbf{x y}=\sum_{j=1}^{m} x_{j} y_{j}, \quad \Pi(\mathbf{x})=\prod_{j=1}^{m}\left(\left|x_{j}\right|+1\right) \\
\Pi_{1}(\mathbf{x})=\prod_{j=1}^{m} x_{j}, \quad d \mathbf{x}=d x_{1} d x_{2} \ldots d x_{m}
\end{gathered}
$$

Будем говорить, что $\mathbf{x} \leqslant \mathbf{y}(\mathbf{x}<\mathbf{y})$, если $x_{j} \leqslant y_{j}\left(x_{j}<y_{j}\right)$ при $j=1,2, \ldots, m$. Если $a$ - число, то через а будем обозначать вектор, все координаты которого равны $a$. Размерность этого вектора каждый раз будет ясна из контекста.

Все рассматриваемые ниже функции предполагаются $2 \pi$-периодическими по каждой переменной. Если $1 \leqslant p<\infty$, то, как обычно, через $L_{p}\left(T^{m}\right)$ обозначается пространство суммируемых в $p$-й степени функций с нормой

$$
\|f(\mathbf{x})\|_{p}=\left(\int_{T^{m}}|f(\mathbf{x})|^{p} d \mathbf{x}\right)^{\frac{1}{p}}
$$

а модуль непрерывности функции $f(\mathbf{x}) \in L_{p}\left(T^{m}\right)-$ это

$$
\omega(f, \delta)_{p}=\sup _{\mathbf{t} \in \mathbb{R}^{m}:|\mathbf{t}|<\delta}\|f(\mathbf{x}+\mathbf{t})-f(\mathbf{x})\|_{p}
$$

Исследования, описанные в данной статье, были выполнены при финансовой поддержке Российского фонда фундаментальных исследований (проект № 97-01-00010).

(C) М.И. Дьяченко, 1998 
Если $f(\mathbf{x}) \in L\left(T^{m}\right)$, то

$$
\sum_{\mathbf{n} \in \mathbb{Z}^{m}} a_{\mathbf{n}}(f) e^{i \mathbf{n} \mathbf{x}}, \quad a_{\mathbf{n}}(f)=\frac{1}{(2 \pi)^{m}} \int_{T^{m}} f(\mathbf{x}) e^{-i \mathbf{n} \mathbf{x}} d \mathbf{x}
$$

- $m$-мерный тригонометрический ряд Фурье данной функции.

Через $C$ ниже будут обозначаться абсолютные положительные постоянные (не обязательно одинаковые в различных случаях), через $C(m)$ - положительные постоянные, зависяшие лишь от размерности пространства, и т.д.

Хорошо известна следующая теорема Харди-Литтлвуда (по поводу одномерного случая см. [1, с. 165], а многомерный результат может быть получен с помошью индукции).

Tеорема А. а) Если $1<p \leqslant 2$ и функиия $f(\mathbf{x})$ принадлежит $L_{p}\left(T^{m}\right)$, то

$$
J_{p}(f)=\left(\sum_{\mathbf{n} \in \mathbb{Z}^{m}}\left|a_{\mathbf{n}}(f)\right|^{p}(\Pi(\mathbf{n}))^{p-2}\right)^{\frac{1}{p}} \leqslant C(p, m)\|f(\mathbf{x})\|_{p}<\infty .
$$

б) Если $2 \leqslant p<\infty$ и коэффициенты ряда

$$
\sum_{\mathbf{n} \in \mathbb{Z}^{m}} a_{\mathbf{n}} e^{i \mathbf{n x}}
$$

удовлетворяют условию

$$
\sum_{\mathbf{n} \in \mathbb{Z}^{m}}\left|a_{\mathbf{n}}\right|^{p}(\Pi(\mathbf{n}))^{p-2}<\infty
$$

то данный ряд является рядом Фурье функции $f(\mathbf{x}) \in L_{p}\left(T^{m}\right)$.

Кроме того (см. [2, с. 657]), Г.Харди и Дж.Литтлвуд установили, что если $m=1$ и коэффициенты $a_{n} \downarrow 0$ при $n \rightarrow \infty$, то обе части теоремы А справедливы при $1<p<\infty$.

В многомерной ситуации можно давать различные определения монотонности. Наиболее употребительным является понятие монотонности в смысле Харди.

ОПРЕДЕЛЕНИЕ 1. Обозначим через $\Gamma_{m}$ множество всех $m$-мерных векторов из нулей и единиц. Пусть $\left\{a_{\mathbf{n}}\right\}_{\mathbf{n} \in \mathbb{Z}_{+}^{m}}-$ последовательность неотрицательных чисел такая, что

$$
\lim _{\max _{1 \leqslant j \leqslant m} n_{j} \rightarrow \infty} a_{\mathbf{n}}=0,
$$

и для любого $\mathbf{n} \in \mathbb{Z}_{+}^{m}$ имеем

$$
\sum_{\boldsymbol{\gamma} \in \Gamma_{m}} a_{\mathbf{n}+\boldsymbol{\gamma}}(-1)^{\gamma_{1}+\cdots+\gamma_{m}} \geqslant 0 .
$$


Тогда данная последовательность называется монотонной в смысле Харди.

$\Phi$. Морищем [3] было установлено, что упомянутый выше одномерный результат Харди-Литтлвуда о тригонометрических рядах с монотонными коэффищиентами остается справедливым и для кратных рядов, если монотонность понимается в смысле Харди. В то же время ряды с монотонными в смысле Харди коэффициентами образуют достаточно узкий класс. Так, даже среди ядер Дирихле только прямоугольные имеют коэффициенты, монотонные в смысле Харди в каждом координатном квадранте. В связи с этим представляется естественным следующее определение.

ОПРЕДЕЛЕНИЕ 2. Пусть $\left\{a_{\mathbf{n}}\right\}_{\mathbf{n} \in \mathbb{Z}_{+}^{m}}$ - последовательность неотрицательных чисел. Тогда скажем, что эта последовательность монотонно убъвает, если для любых $\mathbf{n}, \mathbf{k} \in \mathbb{Z}_{+}^{m}$ таких, что $\mathbf{n} \leqslant \mathbf{k}$, имеем $a_{\mathbf{n}} \geqslant a_{\mathbf{k}}$.

Автором (см. [4], [5]) были получены следующие многомерные обобщения теоремы Харди-Литтлвуда.

Теорема Б. Пусть $2<p<\infty$, функиия $f(\mathbf{x})$ принадлежит $L_{p}\left(T^{m}\right)$ и ее ряд Фурье имеет вид

$$
\sum_{\mathbf{n} \in \mathbb{Z}_{+}^{m}} a_{\mathbf{n}}(f) e^{i \mathbf{n} \mathbf{x}}
$$

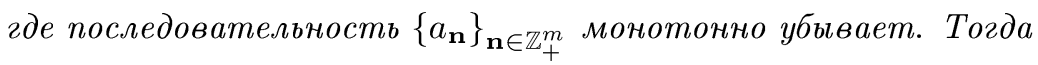

$$
J_{p}(f) \leqslant C(p, m)\|f(\mathbf{x})\|_{p}<\infty
$$

ТЕОРемА В. Если $\frac{2 m}{m+1}<p<2$, коэффициенты т-кратного тригонометрического ряда

$$
\sum_{\mathbf{n} \in \mathbb{Z}_{+}^{m}} a_{\mathbf{n}} e^{i \mathbf{n} \mathbf{x}}
$$

монотонно убивают и

$$
\sum_{\mathbf{n} \in \mathbb{Z}_{+}^{m}} a_{\mathbf{n}}^{p}(\Pi(\mathbf{n}))^{p-2}<\infty
$$

то данный ряд является рядом Фурье функиии $f(\mathbf{x}) \in L_{p}\left(T^{m}\right)$. При этом при $p=\frac{2 m}{m+1}$ утверждение становится неверным.

Далее, автором [6] был предложен к рассмотрению класс кусочно монотонных функций, в определенном смысле двойственный к классу рядов с монотонными коэффициентами. 
ОПРЕДЕЛЕНИЕ 3 . Пусть $\mathbf{a}, \mathbf{b} \in T^{m}, \mathbf{a}<\mathbf{b}$ и $\boldsymbol{\gamma} \in \Gamma_{m}$. Тогда скажем, что функиия $f(\mathbf{x})$ принадлежит $M(\mathbf{a}, \mathbf{b}, \boldsymbol{\gamma})$, если $f(\mathbf{x})=0$ при $\mathbf{x} \in T^{m} \backslash[\mathbf{a}, \mathbf{b}]$ и если для любых $\mathbf{x}, \mathbf{y} \in(\mathbf{a}, \mathbf{b})$ таких, что $(-1)^{\gamma_{j}} x_{j} \leqslant(-1)^{\gamma_{j}} y_{j}$ при $j=1,2, \ldots, m$, имеем $f(\mathbf{x}) \geqslant f(\mathbf{y})$.

ОПРЕДЕЛЕНИЕ 4. Будем говорить, что функиия $f(\mathbf{x})$ принадлежит $P M(f(\mathbf{x})$ кусочно монотонна), если сушествуют такое конечное дизъюнктное разложение

$$
T^{m}=\bigsqcup_{k=1}^{l}[\mathbf{a}(k), \mathbf{b}(k)),
$$

и такие векторы $\gamma(1), \ldots, \gamma(l) \in \Gamma_{m}$, что при $k=1,2, \ldots, l$ функция $f(\mathbf{x}) X_{[\mathbf{a}(k), \mathbf{b}(k))}(\mathbf{x})$ принадлежит $M(\mathbf{a}(k), \mathbf{b}(k), \gamma(k))$, где $X_{E}(\mathbf{x})$ - характеристическая функция множества $E$.

В работе [6] были установлены такие утверждения.

TeOpema Г. Ecлu $1<p<2, \quad f(\mathbf{x}) \in L\left(T^{m}\right) \cap P M u J_{p}(f)<\infty, m o$ $f(\mathbf{x}) \in L_{p}\left(T^{m}\right)$.

Tеорема Д. Если $2<p<2+\frac{1}{m-1}=\frac{2 m-1}{m-1} u f(\mathbf{x}) \in L_{p}\left(T^{m}\right) \cap P M$, то для любого $\varepsilon>0$ имеем $J_{p-\varepsilon}(f)<\infty$. При этом найдется ограниченная кусочно монотонная функиия $f_{m}(\mathbf{x})$ такая, что $J_{2+\frac{1}{m-1}}\left(f_{m}\right)=\infty$.

ЗАмЕчАниЕ 1. Представляется любопытным, что показатель $2+\frac{1}{m-1}$ не является сопряженным к $\frac{2 m}{m+1}$, точнее, он расположен вдвое ближе к числу 2 , чем сопряженный показатель $2+\frac{2}{m-1}$.

ЗАмЕчАниЕ 2. Наряду с кусочно монотонными функциями можно рассматривать и более узкий класс кусочно монотонных функций в смысле Харди (см. [6]). Для них аналог теоремы Харди-Литтлвуда справедлив при всех $p$.

Целью настоящей статьи является получение окончательной оценки в теореме Д.

TeOPEMA. Пусть $2<p<2+\frac{1}{m-1} u f(\mathbf{x}) \in L_{p}\left(T^{m}\right) \cap P M$. Тогдa

$$
J_{p}(f) \leqslant C(p, m, l)\|f(\mathbf{x})\|_{p}<\infty,
$$

где l - число параллелепипедов монотонности функции $f(\mathbf{x})$.

\section{§2. Вспомогательные утверждения}

Установим вначале основную лемму. Метод ее доказательства был разработан В. А. Юдиным [7] в связи с задачей оценки констант Лебега и затем применен автором [5] для получения оценок норм полиномов с монотонными коэффициентами. 
Предварительно введем такое обозначение. Если $f(\mathbf{x}) \in L\left(T^{m}\right)$ и $\mathbf{y} \in \mathbb{R}^{m}$, то положим

$$
\hat{f}(\mathbf{y})=\int_{T^{m}} f(\mathbf{x}) e^{-i \mathbf{y} \mathbf{x}} d \mathbf{x}
$$

Лемма. Пусть $2<p<2+\frac{1}{m-1}$, вектор $\delta=\left(\delta_{1}, \ldots, \delta_{m}\right) \in(0, \pi)^{m}$, вектор $\mathbf{N}=\left(N_{1}, N_{2}, \ldots, N_{m}\right)$, причем $N_{j} \delta_{j} \geqslant 1$ при $j=1,2, \ldots, m, \quad(\mathbf{a}, \mathbf{b}) \subseteq$ $\prod_{j=1}^{m}\left(-\delta_{j}, \delta_{j}\right)$ и для некоторого $\gamma \in \Gamma_{m}$ функция $f(\mathbf{x})$ принадлежит $M(\mathbf{a}, \mathbf{b}, \gamma)$, причем

$$
A=\sup _{\mathbf{x} \in(\mathbf{a}, \mathbf{b})}|f(\mathbf{x})|<\infty
$$

Тогда

$$
\begin{aligned}
I & =\left(\int_{N_{1}}^{\infty} \cdots \int_{N_{m}}^{\infty}\left(\Pi_{1}(\mathbf{x})\right)^{p-2}|\hat{f}(\mathbf{x})|^{p} d \mathbf{x}\right)^{\frac{1}{p}} \\
& \leqslant C(p, m) A\left(\Pi_{1}(\mathbf{N})\right)^{\frac{m-1}{m}-\frac{2 m-1}{m p}}\left(\Pi_{1}(\boldsymbol{\delta})\right)^{\frac{m-1}{m}\left(1-\frac{1}{p}\right)} .
\end{aligned}
$$

ДокАЗАТЕльство. Не ограничивая обшности, будем считать, что $\boldsymbol{\gamma}=\mathbf{0}$. Делая в интеграле

$$
\begin{aligned}
I= & \left(\int_{N_{1}}^{\infty} \ldots \int_{N_{m}}^{\infty}\left(\Pi_{1}(\mathbf{x})\right)^{p-2}\right. \\
& \left.\times\left|\int_{-\delta_{1}}^{\delta_{1}} \cdots \int_{-\delta_{m}}^{\delta_{m}} f\left(t_{1}, \ldots, t_{m}\right) e^{-i\left(t_{1} x_{1}+\cdots+t_{m} x_{m}\right)} d t_{1} \ldots d t_{m}\right|^{p} d x_{1} \ldots d x_{m}\right)^{\frac{1}{p}}
\end{aligned}
$$

замены $y_{1}=\delta_{1} x_{1}, \ldots, y_{m}=\delta_{m} x_{m}$ и $s_{1}=\delta_{1}^{-1} t_{1}, \ldots, s_{m}=\delta_{m}^{-1} t_{m}$, получим

$$
I=\left(\delta_{1} \ldots \delta_{m}\right)^{\frac{1}{p}}\left(\int_{N_{1} \delta_{1}}^{\infty} \ldots \int_{N_{m} \delta_{m}}^{\infty}\left(y_{1} \ldots y_{m}\right)^{p-2}\left|\hat{\psi}\left(y_{1}, \ldots, y_{m}\right)\right|^{p} d y_{1} \ldots d y_{m}\right)^{\frac{1}{p}}
$$

где $\operatorname{supp} \psi \subseteq[-1,1]^{m}$.

Теперь рассмотрим множества

$$
B_{\mathbf{k}}=\prod_{j=1}^{m}\left[2^{k_{j}-1}, 2^{k_{j}}\right)
$$

где $\mathbf{k}=\left(k_{1}, \ldots, k_{m}\right) \geqslant \mathbf{1}$. Пусть

$$
\mathbf{k}(0)=\left(k_{1}(0), \ldots, k_{m}(0)\right)=\left(\left[\log _{2}\left(N_{1} \delta_{1}\right)\right]+1, \ldots,\left[\log _{2}\left(N_{m} \delta_{m}\right)\right]+1\right)
$$


где, как обычно, через $[a]$ обозначается целая часть числа $a$. Тогда

$$
\begin{aligned}
I & \leqslant\left(\delta_{1} \ldots \delta_{m}\right)^{\frac{1}{p}} \sum_{\mathbf{k}=\mathbf{k}(0)}^{\infty}\left(\int_{B_{\mathbf{k}}}\left(y_{1} \ldots y_{m}\right)^{p-2}\left|\hat{\psi}\left(y_{1}, \ldots, y_{m}\right)\right|^{p} d y_{1} \ldots d y_{m}\right)^{\frac{1}{p}} \\
& =\left(\delta_{1} \ldots \delta_{m}\right)^{\frac{1}{p}} \sum_{\mathbf{k}=\mathbf{k}(0)}^{\infty} I(\mathbf{k}) .
\end{aligned}
$$

Для любого $\mathbf{k} \geqslant \mathbf{k}(0)$ обозначим $\nu(\mathbf{k})=\max _{1 \leqslant j \leqslant m} k_{j}$. Если, например, для некоторого $\mathbf{k}$ число $\nu(\mathbf{k})$ равно $k_{r}$, то при $\mathbf{y} \in B_{\mathbf{k}}$, учитьвая монотонность функции $\psi(\mathbf{y})$ на ее носителе по каждой переменной, будем иметь

$$
\begin{aligned}
\left|\hat{\psi}\left(y_{1}, \ldots, y_{m}\right)\right| & \\
& \leqslant \int_{[-1,1]^{m-1}}\left|\int_{-1}^{1} \psi\left(x_{1}, \ldots, x_{m}\right) e^{-i y_{r} x_{r}} d x_{r}\right| d x_{1} \ldots d x_{r-1} d x_{r+1} \ldots d x_{m} \\
& \leqslant \frac{C(m) A}{y_{r}} \leqslant C(m) A 2^{-\nu(\mathbf{k})} .
\end{aligned}
$$

Используя оценку В. А. Юдина (см. [7, с. 404]), устанавливаем, что

$$
\begin{aligned}
I(\mathbf{k}) & \leqslant C(p, m)\left(2^{\left(k_{1}+\cdots+k_{m}\right)} A 2^{-\nu(\mathbf{k})}\right)^{\left(1-\frac{2}{p}\right)}\left(\int_{B_{\mathbf{k}}}|\hat{\psi}(\mathbf{y})|^{2} d \mathbf{y}\right)^{\frac{1}{p}} \\
& \leqslant C(p, m)\left(2^{\left(k_{1}+\cdots+k_{m}\right)} A 2^{-\nu(\mathbf{k})}\right)^{\left(1-\frac{2}{p}\right)}\left(\omega\left(\psi, 2^{-\nu(\mathbf{k})}\right)_{2}\right)^{\frac{2}{p}} .
\end{aligned}
$$

Снова используя монотонность функции $\psi(\mathbf{y})$ на ее носителе по каждой переменной, установим, следуя [5, с. 10], что $\omega(\psi, t)_{2} \leqslant C(m) A t^{\frac{1}{2}}$, а потому

$$
I(\mathbf{k}) \leqslant C(p, m) A 2^{\left(k_{1}+\cdots+k_{m}\right)\left(1-\frac{2}{p}\right)} 2^{-\nu(\mathbf{k})\left(1-\frac{1}{p}\right)} .
$$

Учитывая, что при $2<p<2+\frac{1}{m-1}$ имеем $\frac{1}{m}\left(1-\frac{1}{p}\right)>1-\frac{2}{p}$, получаем отсюда (см. (1)), что при указанных $p$ выполняется оценка

$$
\begin{aligned}
I & \leqslant C(p, m)\left(\delta_{1} \ldots \delta_{m}\right)^{\frac{1}{p}} A \sum_{\mathbf{k}=\mathbf{k}(0)}^{\infty} 2^{\left(k_{1}+\cdots+k_{m}\right)\left(\frac{m-1}{m}-\frac{2 m-1}{m p}\right)} \\
& \leqslant C(p, m) A\left(\delta_{1} \ldots \delta_{m}\right)^{\frac{1}{p}} 2^{\left(k_{1}(0)+\cdots+k_{m}(0)\right)\left(\frac{m-1}{m}-\frac{2 m-1}{m p}\right)} \\
& \leqslant C(p, m) A\left(\delta_{1} \ldots \delta_{m}\right)^{\frac{1}{p}}\left(N_{1} \delta_{1} \ldots N_{m} \delta_{m}\right)\left(\frac{m-1}{m}-\frac{2 m-1}{m p}\right) \\
& =C(p, m) A\left(\Pi_{1}(\mathbf{N})\right)^{\frac{m-1}{m}-\frac{2 m-1}{m p}}\left(\Pi_{1}(\boldsymbol{\delta})\right)^{\frac{m-1}{m}\left(1-\frac{1}{p}\right)}
\end{aligned}
$$

что и требовалось доказать.

Лемма А. Если $1<p<\infty u f(\mathbf{x}) \in L_{p}\left(T^{m}\right), m o$

$$
\int_{T^{m}}\left(\Pi_{1}(\mathbf{x})\right)^{-p}\left(\int_{0}^{x_{1}} \cdots \int_{0}^{x_{m}}|f(\mathbf{t})| d \mathbf{t}\right)^{p} d \mathbf{x} \leqslant C(p, m)\|f(\mathbf{x})\|_{p}^{p} .
$$

Это утверждение для $m=1$ доказано в [2, c. 899]. С помощью индукции легко установить справедливость утверждения леммы А для произвольного $m$, что и было сделано автором в статье [4]. 


\section{§3. Доказательство теоремы}

Предварительно докажем следующее утверждение.

ПРЕДЛОЖЕНИЕ 1. Пусть число р и функиия $f(\mathbf{x})$ удовлетворяют условиям теоремы, вектор $\gamma$ принадлежит $\Gamma_{m}$, а множество $D_{\boldsymbol{\gamma}}$ равно $d_{\gamma_{1}} \times d_{\gamma_{2}} \times$ $\cdots \times d_{\gamma_{m}}$, где $d_{\gamma_{j}}=(-\infty,-1] n p u \gamma_{j}=1 u d_{\gamma_{j}}=[1, \infty)$ nрu $\gamma_{j}=0$. Тогда справедлива оченка

$$
Q_{p}(f, \gamma)=\left(\int_{D_{\gamma}}\left|\Pi_{1}(\mathbf{x})\right|^{p-2}\left|\int_{T^{m}} f(\mathbf{t}) e^{-i \mathbf{t x}} d \mathbf{t}\right|^{p} d \mathbf{x}\right)^{\frac{1}{p}} \leqslant C(m, p, l)\|f(\mathbf{x})\|_{p}
$$

ДокАЗАТЕЛЬСтво. По определению класса $P M$

$$
f(\mathbf{x})=\sum_{r=1}^{l} f_{r}(\mathbf{x})
$$

где $f_{r}(\mathbf{x})=f(\mathbf{x}) X_{[\mathbf{a}(r), \mathbf{b}(r))}(\mathbf{x}) \in M(\mathbf{a}(r), \mathbf{b}(r), \boldsymbol{\beta}(r))$ при $r=1,2, \ldots, l$. Очевидно, что для любого $r$ имеем $\left\|f_{r}(\mathbf{x})\right\|_{p} \leqslant\|f(\mathbf{x})\|_{p}$. При этом можно считать, что $\mathbf{b}(r)-\mathbf{a}(r) \leqslant \mathbf{1}$ для всех $r$. Далее, поскольку при любом $r$

$$
f_{r}(\mathbf{x})=f_{r,+}(\mathbf{x})-f_{r,-}(\mathbf{x})
$$

где

$$
f_{r,+}(\mathbf{x})=\max \left(f_{r}(\mathbf{x}), 0\right), \quad f_{r,-}(\mathbf{x})=\max \left(-f_{r}(\mathbf{x}), 0\right)
$$

и при этом имеем

$$
f_{r,+}(\mathbf{x}),-f_{r,-}(\mathbf{x}) \in M(\mathbf{a}(r), \mathbf{b}(r), \boldsymbol{\beta}(r))
$$

и

$$
\left\|f_{r,+}(\mathbf{x})\right\|_{p},\left\|f_{r,-}(\mathbf{x})\right\|_{p} \leqslant\|f(\mathbf{x})\|_{p}
$$

то дальнейшие рассуждения будем проводить для неотрицательных функций. Кроме того, если $1 \leqslant r \leqslant l$ и $g_{r}(\mathbf{x})=f_{r}(\mathbf{x}-\mathbf{a}(r))$, то $Q_{p}\left(g_{r}, \gamma\right)=Q_{p}\left(f_{r}, \gamma\right)$ при любом $\gamma \in \Gamma_{m}$. Если же $h_{r}(\mathbf{t})=f_{r}\left(t_{1}, \ldots, t_{j-1},-t_{j}, t_{j+1}, \ldots, t_{m}\right)$ при некотором $j$, то $Q_{p}\left(h_{r}, \gamma\right)=Q_{p}\left(f_{r}, \gamma^{\prime}\right)$, где $\gamma_{k}^{\prime}=\gamma_{k}$ при $k \neq j$ и $\gamma_{j}^{\prime}=1-\gamma_{j}$. Исходя из изложенного вьше, будем считать, что неотрицательная функция $f(\mathbf{x})$ принадлежит $L_{p}\left(T^{m}\right) \cap M(\mathbf{0}, \mathbf{b}, \mathbf{0})$, где $\mathbf{b} \leqslant \mathbf{1}$. Кроме того, будем оценивать лишь $Q_{p}(f)=Q_{p}(f, \mathbf{0})$, поскольку для других $\gamma$ оценки проводятся аналогично. Мы докажем, что в этом случае

$$
Q_{p}(f) \leqslant C(p, m)\|f(\mathbf{x})\|_{p}
$$


Имеем

$$
\begin{aligned}
Q_{p}(f) & \leqslant \sum_{B \subseteq\{1, \ldots, m\}}\left(\int_{[1, \infty)^{m}}\left(\Pi_{1}(\mathbf{x})\right)^{p-2}\left|\int_{A_{B}(\mathbf{x})} f(\mathbf{t}) e^{-i \mathbf{t x}} d \mathbf{t}\right|^{p} d \mathbf{x}\right)^{\frac{1}{p}} \\
& =\sum_{B \subseteq\{1, \ldots, m\}} Q_{p, B}(f),
\end{aligned}
$$

где

$$
\begin{gathered}
A_{B}(\mathbf{x})=\prod_{j=1}^{m} \Delta(j, B, \mathbf{x}), \\
\Delta(j, B, \mathbf{x})=\left\{\begin{array}{l}
{\left[0,2^{-k}\right], \text { если } j \in B \text { и } 2^{k-1} \leqslant x_{j}<2^{k}, \text { где } k=1,2, \ldots,} \\
{\left[2^{-k}, 1\right], \text { если } j \notin B \text { и } 2^{k-1} \leqslant x_{j}<2^{k}, \text { где } k=1,2, \ldots}
\end{array}\right.
\end{gathered}
$$

Поскольку все $Q_{p, B}(f)$ оцениваются одинаково, проведем рассуждения для $B=$ $\{1, \ldots, k\}$, где $k \leqslant m$, и для $B=\varnothing$ (в этом случае считаем, что $k=0)$. Используя лемму А, получим

$$
\begin{aligned}
& \left(Q_{p, B}\right)^{p}=\int_{[1, \infty)^{m}}\left(\Pi_{1}(\mathbf{x})\right)^{p-2} \mid \int_{\Delta(1, B, \mathbf{x})} \cdots \int_{\Delta(k, B, \mathbf{x})} \\
& \times\left.\left(\int_{\Delta(k+1, B, \mathbf{x})} \cdots \int_{\Delta(m, B, \mathbf{x})} f(\mathbf{t}) e^{-i \mathbf{t x}} d t_{k+1} \ldots d t_{m}\right) d t_{1} \ldots d t_{k}\right|^{p} d \mathbf{x} \\
& \leqslant \int_{[1, \infty)^{m-k}}\left(x_{k+1} \ldots x_{m}\right)^{p-2} \int_{[1, \infty)^{k}}\left(x_{1} \ldots x_{k}\right)^{p-2} \\
& \times\left(\int_{0}^{\frac{1}{x_{1}}} \cdots \int_{0}^{\frac{1}{x_{k}}} \mid \int_{\Delta(k+1, B, \mathbf{x})} \cdots \int_{\Delta(m, B, \mathbf{x})} f(\mathbf{t})\right. \\
& \left.\times e^{-i \mathbf{t x}} d t_{k+1} \ldots d t_{m} \mid d t_{1} \ldots d t_{k}\right)^{p} d x_{1} \ldots d x_{k} d x_{k+1} \ldots d x_{m} \\
& =\int_{[1, \infty)^{m-k}}\left(x_{k+1} \ldots x_{m}\right)^{p-2} \int_{(0,1]^{k}}\left(u_{1} \ldots u_{k}\right)^{-p} \\
& \times\left(\int_{0}^{u_{1}} \cdots \int_{0}^{u_{k}} \mid \int_{\Delta(k+1, B, \mathbf{x})} \cdots \int_{\Delta(m, B, \mathbf{x})} f(\mathbf{t})\right. \\
& \left.\times e^{-i\left(t_{k+1} x_{k+1}+\cdots+t_{m} x_{m}\right)} d t_{k+1} \ldots d t_{m} \mid d t_{1} \ldots d t_{k}\right)^{p} d u_{1} \ldots d u_{k} d x_{k+1} \ldots d x_{m} \\
& \leqslant C(p, m) \int_{[1, \infty)^{m-k}}\left(x_{k+1} \ldots x_{m}\right)^{p-2} \\
& \times \int_{(0,1]^{k}} \mid \int_{\Delta(k+1, B, \mathbf{x})} \cdots \int_{\Delta(m, B, \mathbf{x})} f(\mathbf{t}) \\
& \times\left. e^{-i\left(t_{k+1} x_{k+1}+\cdots+t_{m} x_{m}\right)} d t_{k+1} \ldots d t_{m}\right|^{p} d t_{1} \ldots d t_{k} d x_{k+1} \ldots d x_{m}
\end{aligned}
$$




$$
\begin{aligned}
= & C(p, m) \int_{(0,1]^{k}} \int_{[1, \infty)^{m-k}}\left(x_{k+1} \ldots x_{m}\right)^{p-2} \\
& \times \mid \int_{\Delta(k+1, B, \mathbf{x})} \cdots \int_{\Delta(m, B, \mathbf{x})} f(\mathbf{t}) \\
& \times\left. e^{-i\left(t_{k+1} x_{k+1}+\cdots+t_{m} x_{m}\right)} d t_{k+1} \ldots d t_{m}\right|^{p} d x_{k+1} \ldots d x_{m} d t_{1} \ldots d t_{k} \\
\equiv & C(p, m) \int_{(0,1]^{k}} F\left(t_{1}, \ldots, t_{k}\right) d t_{1} \ldots d t_{k} .
\end{aligned}
$$

Отметим, что при $k=m$ требуемая оценка уже получена. Если же $k<m$, то для любых фиксированных $t_{1}, \ldots, t_{k}$ рассмотрим функцию

$$
\begin{aligned}
F\left(t_{1}, \ldots, t_{k}\right)= & \sum_{r_{k+1}=1}^{\infty} \ldots \sum_{r_{m}=1}^{\infty} \int_{2^{r_{k+1}-1}}^{2^{r_{k+1}}} \ldots \int_{2^{r_{m}-1}}^{2^{r_{m}}}\left(x_{k+1} \ldots x_{m}\right)^{p-2} \\
& \times \mid \int_{\Delta(k+1, B, \mathbf{x})} \cdots \int_{\Delta(m, B, \mathbf{x})} f(\mathbf{t}) \\
& \times\left. e^{-i\left(t_{k+1} x_{k+1}+\cdots+t_{m} x_{m}\right)} d t_{k+1} \ldots d t_{m}\right|^{p} d x_{k+1} \ldots d x_{m} \\
\equiv & \sum_{r_{k+1}=1}^{\infty} \cdots \sum_{r_{m}=1}^{\infty} F\left(t_{1}, \ldots, t_{k}, r_{k+1}, \ldots, r_{m}\right) .
\end{aligned}
$$

Применяя неравенство Минковского и лемму, получим, что при любых фиксированных $r_{k+1}, \ldots, r_{m} \geqslant 1$ имеем

$$
\begin{aligned}
& \left(F\left(t_{1}, \ldots, t_{k}, r_{k+1}, \ldots, r_{m}\right)\right)^{\frac{1}{p}} \\
& \leqslant \sum_{l_{k+1}=0}^{r_{k+1}-1} \cdots \sum_{l_{m}=0}^{r_{m}-1}\left(\int_{2^{r_{k+1}-1}}^{2^{r_{k+1}}} \cdots \int_{2^{r_{m}-1}}^{2^{r_{m}}}\left(x_{k+1} \ldots x_{m}\right)^{p-2}\right. \\
& \quad \times \mid \int_{2^{-l_{k+1}-1}}^{2^{-l_{k+1}}} \cdots \int_{2^{-l_{m}-1}}^{2^{-l_{m}}} f(\mathbf{t}) \\
& \left.\quad \times\left. e^{-i\left(t_{k+1} x_{k+1}+\cdots+t_{m} x_{m}\right)} d t_{k+1} \ldots d t_{m}\right|^{p} d x_{k+1} \ldots d x_{m}\right)^{\frac{1}{p}} \\
& \leqslant \\
& \quad C(p, m) \sum_{l_{k+1}=0}^{r_{k+1}-1} \sum_{l_{m}=0}^{r_{m}-1} f\left(t_{1}, \ldots, t_{k}, 2^{-l_{k+1}-1}, \ldots, 2^{-l_{m}-1}\right) \\
& \quad \times 2^{\left(r_{k+1}+\cdots+r_{m}\right)\left(\frac{m-k-1}{m-k}-\frac{2(m-k)-1}{(m-k) p}\right)_{2}} 2^{-\left(l_{k+1}+\cdots+l_{m}\right)\left(1-\frac{1}{p}\right) \frac{m-k-1}{m-k}} .
\end{aligned}
$$


Отсюда (см. (4)) получаем, что

$$
\begin{aligned}
F\left(t_{1}, \ldots, t_{k}\right) \leqslant & C(p, m) \sum_{r_{k+1}=1}^{\infty} \cdots \sum_{r_{m}=1}^{\infty} 2^{\left(r_{k+1}+\cdots+r_{m}\right) p\left(\frac{m-k-1}{m-k}\left(1-\frac{1}{p}\right)-\frac{1}{p}\right)} \\
\times & \left(\sum_{l_{k+1}=0}^{r_{k+1}-1} \cdots \sum_{l_{m}=0}^{r_{m}-1} f\left(t_{1}, \ldots, t_{k}, 2^{-l_{k+1}-1}, \ldots, 2^{-l_{m}-1}\right)\right. \\
& \left.\times 2^{-\left(l_{k+1}+\cdots+l_{m}\right)\left(1-\frac{1}{p}\right) \frac{m-k-1}{m-k}}\right)^{p} .
\end{aligned}
$$

Возьмем $\alpha>0$ таким, чтобы $\alpha<\frac{2 m-1}{m}\left(\frac{1}{p}-\frac{m-1}{2 m-1}\right)$. Тогда поскольку при любом $0 \leqslant k<m$ имеем $\left(1-\frac{1}{p}\right) \frac{m-k-1}{m-k}-\frac{1}{p}+\alpha<0$, то

$$
\begin{aligned}
& F\left(t_{1}, \ldots, t_{k}\right) \leqslant C(p, m) \sum_{r_{k+1}=1}^{\infty} \cdots \sum_{r_{m}=1}^{\infty} 2^{\left(r_{k+1}+\cdots+r_{m}\right) p\left(\frac{m-k-1}{m-k}\left(1-\frac{1}{p}\right)-\frac{1}{p}\right)} \\
& \times\left(\sum_{l_{k+1}=0}^{r_{k+1}-1} \cdots \sum_{l_{m}=0}^{r_{m}-1} f\left(t_{1}, \ldots, t_{k}, 2^{-l_{k+1}-1}, \ldots, 2^{-l_{m}-1}\right)\right. \\
& \left.\times 2^{-\left(l_{k+1}+\cdots+l_{m}\right)\left(\left(1-\frac{1}{p}\right) \frac{m-k-1}{m-k}+\alpha\right)} 2^{\left(l_{k+1}+\cdots+l_{m}\right) \alpha}\right)^{p} \\
& \leqslant C(p, m) \sum_{r_{k+1}=1}^{\infty} \cdots \sum_{r_{m}=1}^{\infty} 2^{\left(r_{k+1}+\cdots+r_{m}\right) p\left(\frac{m-k-1}{m-k}\left(1-\frac{1}{p}\right)-\frac{1}{p}\right)} 2^{\left(r_{k+1}+\cdots+r_{m}\right) p \alpha} \\
& \times \sum_{l_{k+1}=0}^{r_{k+1}-1} \cdots \sum_{l_{m}=0}^{r_{m}-1}\left(f\left(t_{1}, \ldots, t_{k}, 2^{-l_{k+1}-1}, \ldots, 2^{-l_{m}-1}\right)\right)^{p} \\
& \times 2^{-\left(l_{k+1}+\cdots+l_{m}\right) p\left(\left(1-\frac{1}{p}\right) \frac{m-k-1}{m-k}+\alpha\right)} \\
& =C(p, m) \sum_{l_{k+1}=0}^{\infty} \cdots \sum_{l_{m}=0}^{\infty}\left(f\left(t_{1}, \ldots, t_{k}, 2^{-l_{k+1}-1}, \ldots, 2^{-l_{m}-1}\right)\right)^{p} \\
& \times 2^{-\left(l_{k+1}+\cdots+l_{m}\right) p\left(\left(1-\frac{1}{p}\right) \frac{m-k-1}{m-k}+\alpha\right)} \\
& \times \sum_{r_{k+1}=l_{k+1}+1}^{\infty} \cdots \sum_{r_{m}=l_{m}+1}^{\infty} 2^{\left(r_{k+1}+\cdots+r_{m}\right) p\left(\frac{m-k-1}{m-k}\left(1-\frac{1}{p}\right)-\frac{1}{p}+\alpha\right)} \\
& \leqslant C(p, m) \sum_{l_{k+1}=0}^{\infty} \cdots \sum_{l_{m}=0}^{\infty}\left(f\left(t_{1}, \ldots, t_{k}, 2^{-l_{k+1}-1}, \ldots, 2^{-l_{m}-1}\right)\right)^{p} \\
& \times 2^{-\left(l_{k+1}+\cdots+l_{m}\right) p\left(\left(1-\frac{1}{p}\right) \frac{m-k-1}{m-k}+\alpha-\left(1-\frac{1}{p}\right) \frac{m-k-1}{m-k}+\frac{1}{p}-\alpha\right)} \\
& =C(p, m) \sum_{l_{k+1}=0}^{\infty} \cdots \sum_{l_{m}=0}^{\infty}\left(f\left(t_{1}, \ldots, t_{k}, 2^{-l_{k+1}-1}, \ldots, 2^{-l_{m}-1}\right)\right)^{p} 2^{-\left(l_{k+1}+\cdots+l_{m}\right)}
\end{aligned}
$$




$$
\leqslant C(p, m) \int_{[0,1]^{m-k}}\left(f\left(t_{1}, \ldots, t_{k}, t_{k+1}, \ldots, t_{m}\right)\right)^{p} d t_{k+1} \ldots d t_{m}
$$

Поэтому (см. (3) и (5))

$$
\left(Q_{p, B}\right)^{p} \leqslant C(p, m) \int_{(\mathbf{0}, \mathbf{b})}(f(\mathbf{t}))^{p} d \mathbf{t}
$$

откуда (см. (2))

$$
Q_{p}(f) \leqslant C(p, m)\|f(\mathbf{x})\|_{p},
$$

что, как было отмечено выше, эквивалентно доказываемому предложению.

СледСТвиЕ. Пусть выполнены условия предложения 1. Тогда справедлива оченка

$$
J_{p, \boldsymbol{\gamma}}(f)=\left(\sum_{\mathbf{n} \in \mathbb{Z}^{m} \cap D_{\boldsymbol{\gamma}}}\left|a_{\mathbf{n}}(f)\right|^{p}(\Pi(\mathbf{n}))^{p-2}\right)^{\frac{1}{p}} \leqslant C(p, m, l)\|f(\mathbf{x})\|_{p} .
$$

ДоКАЗАТЕЛЬСТвО. Снова рассмотрим только $J_{p, \mathbf{0}}(f)$, поскольку оценки остальных величин проводятся точно так же. Отметим, что достаточно доказать, что если функция $f(\mathbf{x})$ принадлежит $L_{p}\left(T^{m}\right) \cap M(\mathbf{a}, \mathbf{b}, \boldsymbol{\beta})$, где $\mathbf{a}, \mathbf{b} \in T^{m}$ и $\boldsymbol{\beta} \in \Gamma_{m}$, а $2<p<2+\frac{1}{m-1}$, то

$$
J_{p, \mathbf{0}}(f) \leqslant C(p, m)\|f(\mathbf{x})\|_{p} .
$$

Для любого целочисленного вектора $\mathbf{n} \geqslant \mathbf{1}$ пусть $I_{\mathbf{n}}=[\mathbf{n}, \mathbf{n}+\mathbf{1}]$. Тогда имеем

$$
\begin{aligned}
(2 \pi)^{m} a_{\mathbf{n}}(f) & =\int_{(\mathbf{a}, \mathbf{b})} f(\mathbf{t}) e^{-i \mathbf{n} \mathbf{t}} d \mathbf{t}=\int_{(\mathbf{a}, \mathbf{b})} f(\mathbf{t}) \int_{I_{n}} e^{-i \mathbf{t u} \mathbf{u}} d \mathbf{u} \prod_{j=1}^{m} \frac{t_{j} e^{-i \frac{t_{j}}{2}}}{2 \sin \frac{t_{j}}{2}} d \mathbf{t} \\
& =\int_{I_{n}}\left(\int_{(\mathbf{a}, \mathbf{b})} f(\mathbf{t}) \prod_{j=1}^{m}\left(\frac{t_{j}}{2 \operatorname{tg} \frac{t_{j}}{2}}+i \frac{t_{j}}{2}\right) e^{-i \mathbf{t u} \mathbf{u}} d \mathbf{t}\right) d \mathbf{u} \\
& \equiv \int_{I_{n}} \int_{(\mathbf{a}, \mathbf{b})} f(\mathbf{t}) \prod_{j=1}^{m} \psi\left(t_{j}\right) e^{-i \mathbf{t u}} d \mathbf{t} d \mathbf{u .}
\end{aligned}
$$

Отсюда

$$
\begin{aligned}
J_{p, \mathbf{0}}(f) & =\left(\sum_{\mathbf{n} \geqslant \mathbf{1}}(\Pi(\mathbf{n}))^{p-2}\left|\int_{I_{n}} \int_{(\mathbf{a}, \mathbf{b})} f(\mathbf{t}) \prod_{j=1}^{m} \psi\left(t_{j}\right) e^{-i \mathbf{t u}} d \mathbf{t} d \mathbf{u}\right|^{p}\right)^{\frac{1}{p}} \\
& \leqslant\left(\sum_{\mathbf{n} \geqslant \mathbf{1}}(\Pi(\mathbf{n}))^{p-2} \int_{I_{n}}\left|\int_{(\mathbf{a}, \mathbf{b})} f(\mathbf{t}) \prod_{j=1}^{m} \psi\left(t_{j}\right) e^{-i \mathbf{t u}} d \mathbf{t}\right|^{p} d \mathbf{u}\right)^{\frac{1}{p}} \\
& \leqslant\left(\int_{[1, \infty)^{m}}(\Pi(\mathbf{u}))^{p-2}\left|\int_{(\mathbf{a}, \mathbf{b})} f(\mathbf{t}) \prod_{j=1}^{m} \psi\left(t_{j}\right) e^{-i \mathbf{t u}} d \mathbf{t}\right|^{p} d \mathbf{u}\right)^{\frac{1}{p}} .
\end{aligned}
$$


Теперь фиксируем некоторое натуральное $N$ и для $r=1,2, \ldots$ рассмотрим функции

$$
\psi_{r}(\tau)=\sum_{k=1}^{r} \psi\left(-\pi+\frac{2 \pi k}{r}\right) X_{\left[-\pi+\frac{2 \pi(k-1)}{r},-\pi+\frac{2 \pi k}{r}\right)}(\tau) .
$$

Функция $\psi(\tau)$ непрерывна на $\bar{T}$. Поэтому функции $\psi_{r}\left(t_{1}\right)$ при $r \rightarrow \infty$ равномерно на $T$ сходятся к функции $\psi\left(t_{1}\right)$ и ограничены в совокупности. Используя теорему Лебега о предельном переходе, преобразование Абеля и неравенство Минковского, получим

$$
\begin{aligned}
S_{p, \mathbf{0}, N} & (f, 1) \equiv\left(\int_{[1, N]^{m}}(\Pi(\mathbf{u}))^{p-2}\left|\int_{(\mathbf{a}, \mathbf{b})} f(\mathbf{t}) \psi\left(t_{1}\right) e^{-i \mathbf{t u}} d \mathbf{t}\right|^{p} d \mathbf{u}\right)^{\frac{1}{p}} \\
= & \lim _{r \rightarrow \infty}\left(\int_{[1, N]^{m}}(\Pi(\mathbf{u}))^{p-2}\left|\int_{(\mathbf{a}, \mathbf{b})} f(\mathbf{t}) \psi_{r}\left(t_{1}\right) e^{-i \mathbf{t u}} d \mathbf{t}\right|^{p} d \mathbf{u}\right)^{\frac{1}{p}} \\
= & \lim _{r \rightarrow \infty}\left(\int_{[1, N]^{m}}(\Pi(\mathbf{u}))^{p-2} \mid \int_{(\mathbf{a}, \mathbf{b})} f(\mathbf{t})\right. \\
& \times\left(\sum_{k=1}^{r-1}\left(\psi\left(-\pi+\frac{2 \pi k}{r}\right)-\psi\left(-\pi+\frac{2 \pi(k+1)}{r}\right)\right)\right. \\
& \left.\left.\times X_{\left[-\pi,-\pi+\frac{2 \pi k}{r}\right)}\left(t_{1}\right)+\psi(\pi) X_{[-\pi, \pi)}\left(t_{1}\right)\right)\left.e^{-i \mathbf{t u}} d \mathbf{t}\right|^{p} d \mathbf{u}\right)^{\frac{1}{p}} \\
\leqslant & \lim _{r \rightarrow \infty}\left(\sum_{k=1}^{r-1}\left|\psi\left(-\pi+\frac{2 \pi k}{r}\right)-\psi\left(-\pi+\frac{2 \pi(k+1)}{r}\right)\right|^{+|\psi(\pi)|)}\right. \\
& \times \sup _{k}\left(\int_{[1, N]^{m}}(\Pi(\mathbf{u}))^{p-2}\left|\int_{(\mathbf{a}, \mathbf{b})} f(\mathbf{t}) X_{\left[-\pi,-\pi+\frac{2 \pi k}{r}\right)}\left(t_{1}\right) e^{-i \mathbf{t u}} d \mathbf{t}\right|^{p} d \mathbf{u}\right)^{\frac{1}{p}} .
\end{aligned}
$$

Действительная часть функции $\psi\left(t_{1}\right)$ возрастает на $[-\pi, 0]$ и убывает на $[0, \pi]$, а мнимая часть этой функции монотонно возрастает на $T$. Поэтому

$$
\sum_{k=1}^{r-1}\left|\psi\left(-\pi+\frac{2 \pi k}{r}\right)-\psi\left(-\pi+\frac{2 \pi(k+1)}{r}\right)\right|+|\psi(\pi)| \leqslant C
$$

Далее, при $k=1,2, \ldots, r$ функция вида $f(\mathbf{t}) X_{\left[-\pi,-\pi+\frac{2 \pi k}{r}\right)}\left(t_{1}\right)$ принадлежит $M(\mathbf{a}, \mathbf{d}, \boldsymbol{\beta})$, где $d_{1}=\min \left(b_{1},-\pi+\frac{2 \pi k}{r}\right)$ и $d_{j}=b_{j}$ при $j=2,3, \ldots, m$. Тогда согласно предложению 1 имеем

$$
\begin{aligned}
& \left(\int_{[1, N]^{m}}(\Pi(\mathbf{u}))^{p-2}\left|\int_{(\mathbf{a}, \mathbf{b})} f(\mathbf{t}) X_{\left[-\pi,-\pi+\frac{2 \pi k}{r}\right)}\left(t_{1}\right) e^{-i \mathbf{t u} \mathbf{u}} d \mathbf{t}\right|^{p} d \mathbf{u}\right)^{\frac{1}{p}} \\
& \leqslant C(p, m)\left\|f(\mathbf{x}) X_{\left[-\pi,-\pi+\frac{2 \pi k}{r}\right)}\left(x_{1}\right)\right\|_{p}
\end{aligned}
$$


Отсюда (см. (8) и (9))

$$
S_{p, \mathbf{0}, N}(f, 1) \leqslant \sup _{k, r} C(p, m)\left\|f(\mathbf{x}) X_{\left[-\pi,-\pi+\frac{2 \pi k}{r}\right)}\left(x_{1}\right)\right\|_{p} \leqslant C(p, m)\|f(\mathbf{x})\|_{p}
$$

В силу произвольности числа $N$ отсюда вытекает

$$
\left(\int_{[1, \infty)^{m}}(\Pi(\mathbf{u}))^{p-2}\left|\int_{(\mathbf{a}, \mathbf{b})} f(\mathbf{t}) \psi\left(t_{1}\right) e^{-i \mathbf{t u}} d \mathbf{t}\right|^{p} d \mathbf{u}\right)^{\frac{1}{p}} \leqslant C(p, m)\|f(\mathbf{x})\|_{p} .
$$

Далее, используя это неравенство и повторяя предыдушие рассуждения, получим

$$
\left(\int_{[1, \infty)^{m}}(\Pi(\mathbf{u}))^{p-2}\left|\int_{(\mathbf{a}, \mathbf{b})} f(\mathbf{t}) \psi\left(t_{1}\right) \psi\left(t_{2}\right) e^{-i \mathbf{t u}} d \mathbf{t}\right|^{p} d \mathbf{u}\right)^{\frac{1}{p}} \leqslant C(p, m)\|f(\mathbf{x})\|_{p}
$$

Применив последовательно этот метод для всех переменных и учитьвая оценку (7), устанавливаем утверждение следствия.

Перейдем теперь непосредственно к доказательству теоремы. Проведем его с помощью индукции по размерности пространства. Для $m=1$ утверждение теоремы сразу вытекает из доказанного выше следствия (впрочем для $m=1$ теорема была установлена автором в [6]). Предположим, что оценка теоремы уже установлена для размерности $m-1$, где $m \geqslant 2$. Установим требуемую оценку для функции $f(\mathbf{x}) \in L_{p}\left(T^{m}\right) \cap M(\mathbf{a}, \mathbf{b}, \boldsymbol{\beta})$, где $\mathbf{a}, \mathbf{b} \in T^{m}$ и $\boldsymbol{\beta} \in \Gamma_{m}$. Отметим, что функция $m-1$ переменных

$$
g\left(t_{1}, \ldots, t_{m-1}\right)=\frac{1}{2 \pi} \int_{T} f\left(t_{1}, \ldots, t_{m-1}, t_{m}\right) d t_{m}
$$

принадлежит $M\left(\left(a_{1}, \ldots, a_{m-1}\right),\left(b_{1}, \ldots, b_{m-1}\right),\left(\gamma_{1}, \ldots, \gamma_{m-1}\right)\right)$ и $2<p<2+$ $\frac{1}{m-1}<2+\frac{1}{m-2}$, а потому по предположению индукции имеем

$$
\begin{aligned}
\left(\sum_{\mathbf{n} \in \mathbb{Z}^{m}: n_{m}=0}\left|a_{\mathbf{n}}(f)\right|^{p}(\Pi(\mathbf{n}))^{p-2}\right)^{\frac{1}{p}} & =\left(\sum_{\mathbf{k} \in \mathbb{Z}^{m-1}}\left|a_{\mathbf{k}}(g)\right|^{p}(\Pi(\mathbf{k}))^{p-2}\right)^{\frac{1}{p}} \\
& \leqslant C(p, m)\|g(\mathbf{x})\|_{p} \leqslant C(p, m)\|f(\mathbf{x})\|_{p} .
\end{aligned}
$$

Суммы по остальным координатным гиперплоскостям оцениваются точно так же, и, учитьвая согласно следствию, что

$$
\left(\sum_{n_{1} \neq 0} \cdots \sum_{n_{m} \neq 0}\left|a_{\mathbf{n}}(f)\right|^{p}(\Pi(\mathbf{n}))^{p-2}\right)^{\frac{1}{p}} \leqslant C(p, m)\|f(\mathbf{x})\|_{p},
$$

получаем утверждение теоремы. 


\section{Список литературы}

1. Зигмунд А. Тригонометрические ряды. Т. 1. М.: Мир, 1965.

2. Бари Н. К. Тригонометрические ряды. М.: Физматгиз, 1961.

3. Moricz F. On double cosine, sine and Walsh series with monotone coefficients // Proc. Amer. Math. Soc. 1990. V. 109. № 2. P. 417-425.

4. Dyachenko M. I. Multiple trigonometric series with lexicographically monotone coefficients // Anal. Math. 1990. V. 16. № 3. P. 173-190.

5. Дьяченко М. И. Нормы ядер Дирихле и некоторых других тригонометрических полиномов в пространствах $L_{p} / /$ Матем. сб. 1993. Т. 184. №3. С. 3-20.

6. Дьяченко М.И. Кусочно-монотонные функции многих переменных и теорема Харди-Литтлвуда // Изв. АН СССР. Сер. матем. 1991. Т. 55. №6. С. 1156-1170.

7. Юдин В. А. Поведение констант Лебега // Матем. заметки. 1975. Т. 17. № 3. С. 401-405.

Поступило в редакцию

12.XI.1996 